Check for updates

Cite this: Phys. Chem. Chem. Phys., $2017,19,27090$

Received 17th July 2017, Accepted 25th September 2017

DOI: $10.1039 / c 7 c p 04811 d$

rsc.li/pccp

\section{DFT study of the structure, chemical ordering and molecular adsorption of Pd-Ir nanoalloys $\dagger$}

\author{
Tian-E Fan, (D) ab ${ }^{\text {Ilker Demiroglu, }}{ }^{\mathrm{b}}$ Heider A. Hussein, ${ }^{\mathrm{bc}}$ Tun-Dong Liu*a and \\ Roy L. Johnston (D)*b
}

\begin{abstract}
The structures and surface adsorption sites of Pd-Ir nanoalloys are crucial to the understanding of their catalytic performance because they can affect the activity and selectivity of nanocatalysts. In this article, density functional theory (DFT) calculations are performed on bare Pd-Ir nanoalloys to systematically explore their stability and chemical ordering properties, before studying the adsorption of $\mathrm{CO}$ on the nanoalloys. First, the structural stability of 38-atom and 79-atom truncated octahedral (TO) Pd-Ir nanoalloys are investigated. Then the adsorption properties and preferred adsorption sites of $\mathrm{CO}$ on 38-atom Pd-Ir nanoalloys are considered. The $\mathrm{Pd}_{\text {shell }} \mathrm{I}_{\text {core }}$ structure, which has the lowest energy of all the considered isomers, exhibits the highest structural stability, while the $\mathrm{Pd}_{\text {core }} \mid \mathrm{r}_{\text {shell }}$ configuration is the least stable. In addition, the adsorption strength of $\mathrm{CO}$ on $\mathrm{Ir}$ atoms is found to be greater than on Pd for $\mathrm{Pd}-\mathrm{Ir}$ nanoclusters. The preferred adsorption sites of $\mathrm{CO}$ on pure Pd and Ir clusters are in agreement with calculations and experiments on extended Pd and Ir surfaces. In addition, d-band center and charge effects on $\mathrm{CO}$ adsorption strength on $\mathrm{Pd}-\mathrm{Ir}$ nanoalloys are analyzed by comparison with pure clusters. The study provides a valuable theoretical insight into catalytically active Pd-Ir nanoalloys.
\end{abstract}

\section{Introduction}

Nanosized clusters or nanoparticles are new materials, which differ from individual atoms and bulk materials; showing unique chemical and physical properties. ${ }^{1}$ Metal nanoclusters are of interest as catalysts due to their high surface-volume ratio and high proportion of low-coordinated active sites. ${ }^{2}$ Among metallic nanoclusters, palladium-iridium (Pd-Ir) nanoalloys have been considered as important catalysts in a number of chemical and physical applications. ${ }^{3-8}$ For example, Pd-Ir nanoalloys act as catalysts for a range of organic reactions, including olefin hydrogenation and tetralin hydro-conversion through selective ring opening. ${ }^{9}$ They are also applied in the preferential oxidation of $\mathrm{CO}$ for the elimination of impurities in $\mathrm{H}_{2}$ production. ${ }^{10}$ Palladium (Pd) is regarded as one of the best catalysts, owing to its excellent reactivity and stability, being the only bulk metal that can form a hydride phase at ambient temperature and pressure. ${ }^{11} \mathrm{Pd}-\mathrm{Ir}$ is a demixing system in the bulk, exhibiting miscibility gaps with relatively high critical temperatures, ${ }^{12,13}$ but miscibility is enhanced at the nanoscale.,

\footnotetext{
${ }^{a}$ Department of Automation, Xiamen University, Xiamen, 361005, China. E-mail: ltd@xmu.edu.cn

${ }^{b}$ School of Chemistry, University of Birmingham, Edgbaston, Birmingham B15 2TT, UK. E-mail: r.l.johnston@bham.ac.uk

${ }^{c}$ Department of Chemistry, College of Science, University of Kufa, Najaf, Iraq

$\dagger$ Electronic supplementary information (ESI) available. See DOI: 10.1039/c7cp04811d
}

Nanoalloying Pd and Ir not only enhances the catalytic activity, due to the synergistic effect of atomic configuration and electronic structures of the component metals, but also improves the selectivity of catalysts. ${ }^{14}$

For Pd-Ir nanoalloys, their (geometric and electronic) structures are crucial to the understanding of their catalytic performances because the activity and selectivity are closely associated with structure. The catalytic performance also depends on composition, surface segregation and chemical ordering, and these characteristics of alloyed NPs can be controlled to tune their optical, electrical, and catalytic properties. ${ }^{15-17}$ Moreover, both theory and experiment show that the binding of ligands can change the chemical ordering and the structures of nanoalloys. ${ }^{18-20}$ The surface sites and the bonding of adsorbates under reaction conditions can also affect the surface structure and change the activity and the selectivity of nanocatalysts. Surface segregation of $\mathrm{Pd}$ has been reported to occur in the presence of reactive gases such as $\mathrm{CO}$ and $\mathrm{O}_{2},{ }^{21,22}$ and the binding of CO molecules is commonly used as a probe to identify the nature of metal surface adsorption sites. ${ }^{23,24} \mathrm{Pd}-\mathrm{Ir}$ nanoalloys are often used as catalysts in the preferential oxidation of CO (PROX). ${ }^{8,10}$

The Pd-Ir system has rarely been studied computationally, ${ }^{25-29}$ and relatively few catalytic studies have been devoted to Pd-Ir nanoalloys. ${ }^{9,27,30}$ In this study, the structure and chemical ordering of bare and CO-adsorbed Pd-Ir nanoalloys have been investigated theoretically by using density functional theory (DFT). The mixing energy and the effect of CO adsorption on 
Pd-Ir nanoalloys have been considered. Since the d-band model $^{31}$ is particularly important for understanding metaladsorbate bond formation and trends in reactivity, the d-band center is calculated to analyze the adsorption strength caused by metal-adsorbate interaction. This article is structured as follows. Section 2 briefly describes the calculation methods. Section 3 presents the calculated results, discussion and comparison with available experimental results for bare Pd-Ir and CO-adsorbed Pd-Ir nanoalloys. The main conclusions are summarized in the fourth section.

\section{Methodology}

\subsection{Model}

In our atomistic calculation, the (fcc packing) truncated octahedron (TO) having six $\{100\}$ and eight $\{111\}$ facets, has been chosen as the nanoparticle model to study 38-atom and 79atom Pd-Ir nanoalloys (Fig. 1). The TO structure is frequently adopted in theoretical and experimental studies, ${ }^{32,33}$ because of its high symmetry $\left(O_{\mathrm{h}}\right)$ and it being a fragment of a face-centered cubic crystal (fcc). The sizes of 38 and 79 atoms have been selected to describe the trend of structural stability for Pd-Ir nanoalloys with different compositions and configurations, which enables predictions for larger experimental fcc-based nanoparticles.

Binary nanoalloys present increasing structural complexity compared with unary nanoclusters because the two components can have variable compositions and exhibit various chemical ordering patterns. ${ }^{14}$ For example, there are ordered, Janus, ballcup, core-shell, sandwich structures and so on in nanoalloys, ${ }^{34}$ some structures are shown in Fig. 2. Here, the preferred position for a single Pd or Ir dopant in 38-atom and 79-atom nanoclusters is first investigated, considering the unique positions for singleatom substitutions. These dopant sites are shown in Fig. 1 for the 79-atom TO.

For other compositions, we have constructed several configurations for 38-atom and 79-atom Pd-Ir nanoalloys. The configurations studied for 38-TO are shown in Fig. 2. The ball-cup structures are generated by embedding (but not completely covering) one

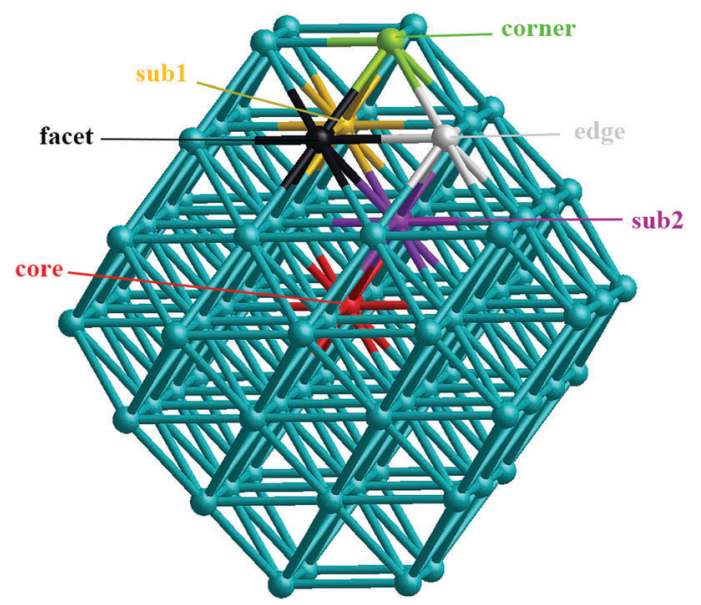

Fig. 1 Unique positions for single-atom doping in 79-atom TO nanoparticles. type of atom in the other. ${ }^{35}$ The Janus alloy particle is formed by dividing the TO structure into discrete Pt and Ir regions, sharing a single (approximately) planar interface. The cluster Janus- $\mathrm{Pd}_{19} \mathrm{Ir}_{19}$ has a perfect half $\mathrm{Pd}$ and half $\mathrm{Ir}$ structure. Ordered structures are created by alternating Pd and Ir layers in the (100) or (111) directions and by occupying different fcc crystal positions with Pd or Ir. The core-shell structures have a core of one metal, completely surrounded by a shell of the other metal. Sandwich structures have a layer of one metal, sandwiched by layers of the other metal. In addition, to compare relative stabilities of different clusters with the same compositions, we also build the hex and centroid structures. For example, hex- $\mathrm{Pd}_{32} \operatorname{Ir}_{6}$ has $6 \mathrm{Ir}$ atoms forming a hexagonal ring surrounding one of the (111) facets of $\mathrm{TO}_{38}$, while centroid$\mathrm{Pd}_{32} \operatorname{Ir}_{6}$ has $6 \mathrm{Ir}$ atoms occupying the centres of 6 (111) facets of TO, in a $D_{3 \mathrm{~d}}$ symmetry arrangement.

For molecular adsorption studies, the CO molecule has been chosen for adsorption on TO 38-atom Pd-Ir nanoalloys. The possible adsorption sites on the surface of TO-shaped nanoparticles are grouped into eight symmetry-inequivalent sites, as shown in Fig. 3. The sites include both (111) and (100) facets. 1 and 2 are the atop sites, on the center of the (111) facet and the edge atom between (111) and (100) facets, respectively. 3, 4 and 5 are bridge sites, respectively on the (111) facet, between the (111) and (100) facets, and between two (111) facets. 6 and 7 are (fcc) and (hcp) hollow sites on the (111) facet, 8 is the hollow site on the (100) facet. Calculations are performed by placing a single CO molecule at each of these sites and carrying out local DFT minimization.

\subsection{DFT calculations}

In this study, all calculations are performed using the DFT method, as implemented in the Vienna ab initio Simulation Package (VASP) code. ${ }^{36}$ The interaction between valence electrons and ionic cores is described by the projector augmented wave (PAW) method. ${ }^{37,38}$ The generalized gradient approximation (GGA) is employed within the Perdew-Burke-Ernzerhof (PBE) parameterization for the exchange-correlation energy functional. ${ }^{39}$ All the calculations are spin-polarized, and the valence electrons are treated explicitly. To avoid spurious periodic interactions, the bare clusters are placed into a sufficiently large supercell to ensure $\sim 10 \AA$ separation by vacuum. All calculated clusters are locally geometrical optimized at the DFT level, where all cluster atoms, are relaxed until the forces on the atoms are lower than $0.01 \mathrm{eV} \AA^{-1}$, and the electronic ground states are determined by requiring a total energy convergence of $10^{-6} \mathrm{eV}$. In order to test the correctness of our computational methodology (VASP/PBE), a comparison of calculated and experimental lattice parameters $(a)$, cohesive energies $\left(E_{\mathrm{c}}\right)$ and bulk modulus $(B)$ for bulk fcc Pd and Ir has been made. As shown in the ESI, $\dagger$ the experimental and calculated values are in good agreement.

\subsection{Energies}

For the stability comparison of Pd-Ir nanoalloys with different compositions and different configurations, a mixing (or excess) 


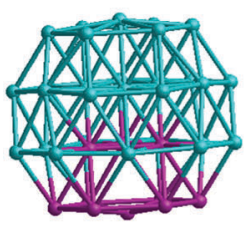

Pd28Ir10 (ball-cup)

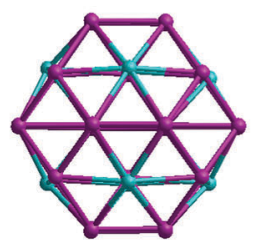

Pd8Ir30 (ordered)

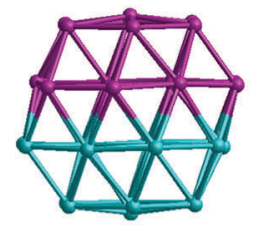

Pd19Ir19

(janus)

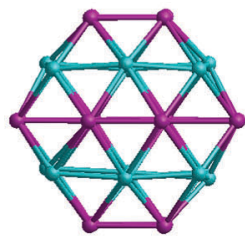

Pd18Ir20 (ordered)
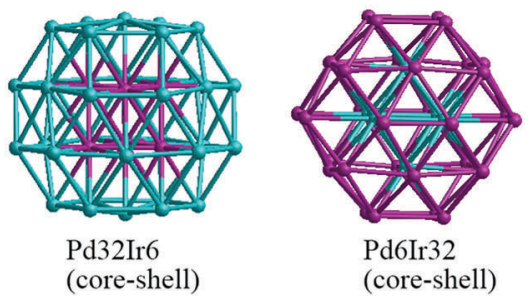

Pd6Ir32

(core-shell)

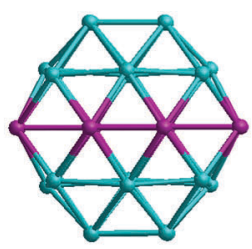

Pd12Ir26

(sandwich)

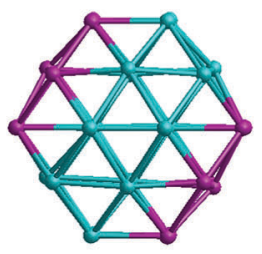

Pd24Ir14 (sandwich)

Fig. 2 38-TO Pd-Ir nanoalloys with different compositions and configurations. In this (and later figures) Pd atoms are shown in blue and Ir atoms in purple.

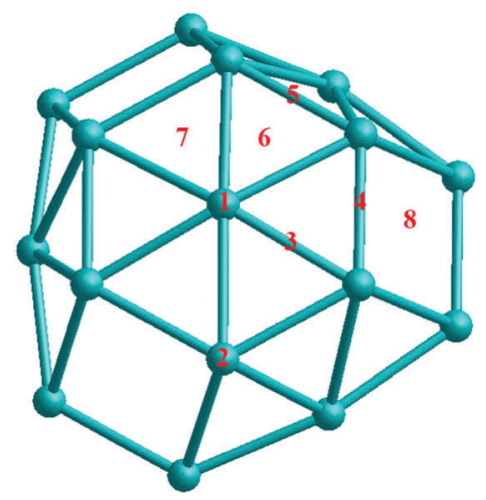

Fig. 3 The eight unique sites for $\mathrm{CO}$ molecular adsorption on the surface of 38-atom TO Pd-Ir nanoalloys.

energy term $(\Delta)$ is calculated using: ${ }^{40}$

$$
\Delta=E_{\mathrm{tot}}\left(\mathrm{Pd}_{m} \mathrm{Ir}_{n}\right)-m \frac{E_{\mathrm{tot}}\left(\mathrm{Pd}_{m+n}\right)}{m+n}-n \frac{E_{\mathrm{tot}}\left(\operatorname{Ir}_{m+n}\right)}{m+n}
$$

where $E_{\text {tot }}\left(\mathrm{Pd}_{m} \mathrm{Ir}_{n}\right)$ denotes the total energy of $\mathrm{Pd}_{m} \mathrm{Ir}_{n}$ nanoalloys, $E_{\text {tot }}\left(\mathrm{Pd}_{m+n}\right)$ and $E_{\text {tot }}\left(\operatorname{Ir}_{m+n}\right)$ are the energies of pure $\mathrm{Pd}$ and Ir clusters with the same size $(m+n)$ as $\operatorname{Pd}_{m} \mathrm{Ir}_{n} . m$ and $n$ are the number of atoms of metal Pd and Ir, respectively. A negative value of mixing energy $(\Delta)$ means an energy decrease after mixing and therefore favorable mixing, whereas positive values indicate a demixing tendency.

The average binding energy $E_{\mathrm{b}}$ is given by:

$$
E_{\mathrm{b}}=\frac{1}{N}\left[m E_{\mathrm{Pd}}+n E_{\mathrm{Ir}}-E\left(\operatorname{Pd}_{m} \operatorname{Ir}_{n}\right)\right]
$$

where $m$ and $n$ are the numbers of Pd and Ir atoms, $E_{\mathrm{Pd}}$ and $E_{\mathrm{Ir}}$ are the electronic energies of a single Pd or Ir atom. $N$ is the total number of atoms, $N=m+n$.

The adsorption energy of a CO molecule on Pd-Ir clusters is calculated as the difference between the total energies of the combined system and separated ones, which is expressed by:

$$
E_{\text {ads }}=E_{\text {tot }}(\text { combined })-E_{\text {tot }}(\text { cluster })-E_{\text {tot }}(\text { adsorbate })
$$

where $E_{\text {tot }}$ (combined) is the total energy of CO adsorbed on the Pd-Ir cluster, $E_{\text {tot }}$ (cluster) is the energy of the locally-minimized bare Pd-Ir cluster and $E_{\text {tot }}$ (adsorbate) is the energy of an isolated CO molecule.

To compare the properties of alloyed clusters and pure clusters, the d-band center is calculated as: ${ }^{28,41}$

$$
d_{\mathrm{center}}=\frac{\int \rho E \mathrm{~d} E}{\rho \mathrm{d} E}
$$

where $\rho$ is the d-band density, $E$ is the d-band energy, $\rho \mathrm{d} E$ is the number of states. The $d_{\text {center }}$ values are calculated only for the (111) facets of the clusters, to allow a comparison between alloyed and pure clusters.

\section{Results and discussion}

\subsection{Bare Pd-Ir nanoalloys}

First, to investigate the site preference for Pd and Ir dopants in the TO Pd-Ir nanoalloys, we calculate the mixing energy of Pd-Ir nanoalloys with only one dopant atom. The results are listed in Table 1. For the 38-TO cluster, a single Ir dopant in the Pd cluster preferentially occupies a core site (having the most negative $\Delta$ value), but it is most unfavourable in the corner (with largest positive 4). Conversely, the most favourable

Table 1 Mixing energies $(\Delta / \mathrm{eV})$ of single dopants in 38-atom and 79 atom TO clusters. In each case, the preferred (most negative $\Delta$ ) sites are indicated in bold and the least stable (most positive 4 ) sites in italics

\begin{tabular}{lccrr}
\hline Position & $\mathrm{Pd}_{37} \mathrm{Ir}_{1}$ & $\mathrm{Pd}_{1} \mathrm{Ir}_{37}$ & $\mathrm{Pd}_{78} \mathrm{Ir}_{1}$ & $\mathrm{Pd}_{1} \operatorname{Ir}_{78}$ \\
\hline Core & $-\mathbf{0 . 4 1 2}$ & 1.170 & $-\mathbf{0 . 1 8 1}$ & 1.596 \\
Facet & -0.055 & 0.318 & 0.216 & 0.292 \\
Corner & 0.449 & $-\mathbf{0 . 0 8 8}$ & 0.803 & $-\mathbf{0 . 3 4 8}$ \\
Edge & - & - & 0.453 & 0.225 \\
Sub1 & - & - & -0.069 & 0.682 \\
Sub2 & - & - & -0.117 & 0.853
\end{tabular}




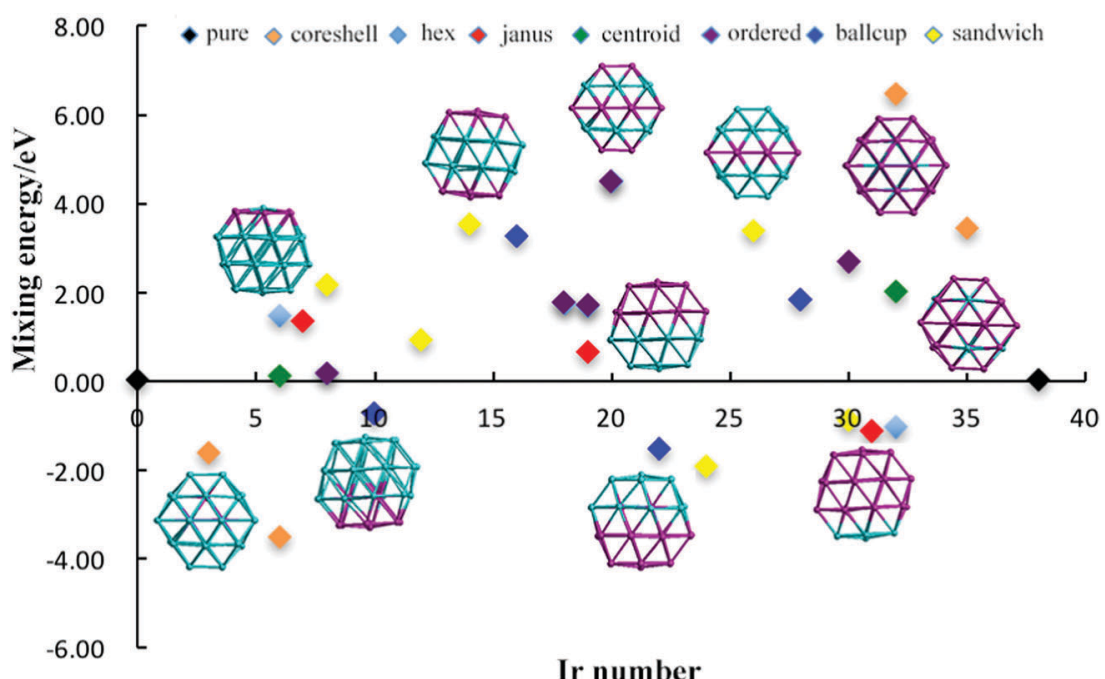

Fig. 4 Mixing energy of 38-TO Pd-Ir nanoalloys with different compositions and configurations. Different colours represent different configurations.

position for a Pd dopant is the corner site, and it is demixing in the core site. The results (in terms of most and least stable sites) for 79-TO are the same as for 38-TO. The stability order for a single Ir dopant in 79-TO Pd clusters is core $>$ subsurface $>$ facet $>$ edge $>$ corner. On the contrary, for a Pd dopant, the stability order is corner $>$ edge $>$ facet $>$ subsurface $>$ core, only the corner position has negative mixing energy, which is similar to the Pd dopant in TO-38 Pd-Ir nanoalloys.

The observed dopant site preferences are in agreement with the higher cohesive energy of Ir $(6.93 \mathrm{eV})$ than Pd $(3.94 \mathrm{eV}) ;^{42}$ the fact that the metal-metal bond strengths are in the order Ir-Ir > Ir-Pd > Pd-Pd $;^{43}$ and the lower (111) surface energy of Pd (0.824 eV per atom) compared to Ir (1.225 eV per atom) ${ }^{44}$

The relative structural stabilities of Pd-Ir nanoalloys is determined by calculating the mixing energies for different compositions and configurations, as shown in Fig. 4 and 5. Fig. 4 describes the variation of mixing energies of 38-atom Pd-Ir nanoalloys with increasing Ir composition. From this figure, one can see that the $\mathrm{Pd}_{\text {shell }} \mathrm{Ir}_{\text {core }}$ structure is the most stable configuration of all the isomers considered (having the most negative) mixing energy, because the Pd atoms on the surface are beneficial to lowering the total energy of Pd-Ir nanoalloys. This is consistent with the results for single dopants in Pd-Ir clusters, since Ir atoms preferentially occupy core sites. Conversely, the $\mathrm{Pd}_{\text {core }} \mathrm{Ir}_{\text {shell }}$ isomer is the least stable structure, which is also consistent with the site preference of Pd doping, which is unfavourable in core sites. In addition, for different chemical orderings of Pd-Ir nanoalloys with the same composition, taking hex-Pd ${ }_{32} \mathrm{Ir}_{6}$, centroid-Pd ${ }_{32} \mathrm{Ir}_{6}$ and core-shell- $\mathrm{Pd}_{32} \mathrm{Ir}_{6}$ nanoalloys as examples, one can see the mixing energy $(\Delta)$ of hex $>$ centroid $>$ core-shell structures, which means for the same compositions, the stability of core-shell $>$ centroid $>$ hex. Similarly, the stability of Janus- $\mathrm{Pd}_{19} \mathrm{Ir}_{19}$ is higher than that of ordered$\mathrm{Pd}_{19} \mathrm{Ir}_{19}$, because of the much higher strength of the Ir-Ir bonds.

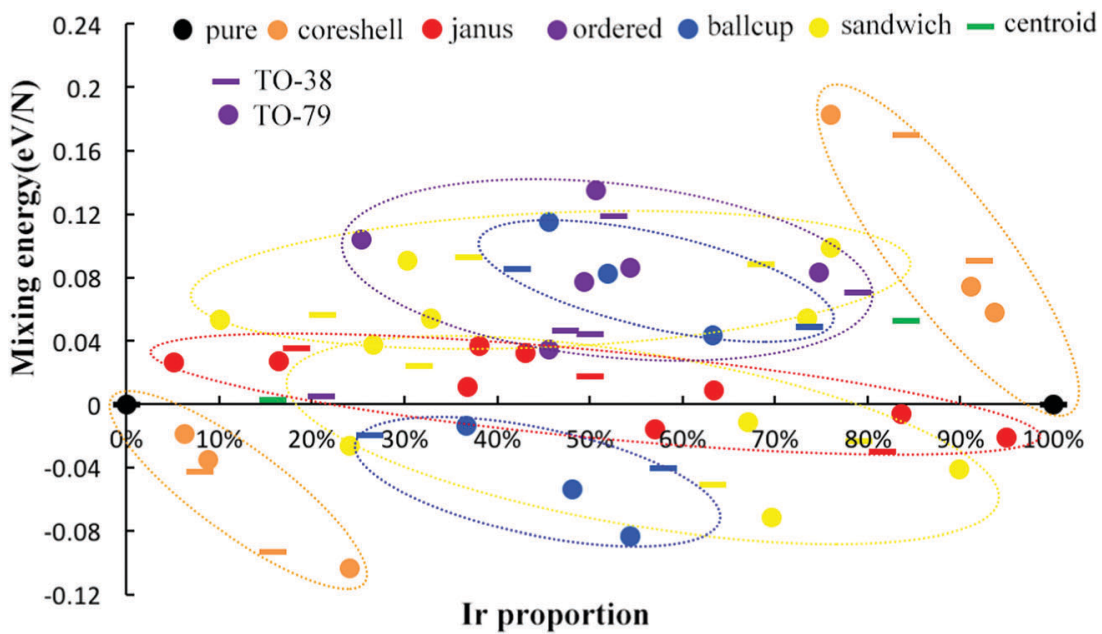

Fig. 5 Comparison of mixing energy per atom for 38-TO and 79-TO Pd-Ir nanoalloys with different compositions and configurations. Different colors represent different configurations, the bar symbol denotes the 38-TO Pd-Ir system, and the circle symbol denotes the 79-TO Pd-Ir system. 
Moreover, for the Janus-type configurations with different compositions, Ir-rich clusters are most stable among all considered compositions. For ordered-structures, the Pd-rich cluster has the lowest mixing energy $(0.176 \mathrm{eV})$ among all ordered structures. Interestingly, for both sandwich-type and ball-cup-type arrangements, the slightly Ir-rich structures have lowest mixing energies with mixing energies of $-1.904 \mathrm{eV}$ and $-1.525 \mathrm{eV}$, respectively.

To investigate the structural stability of Pd-Ir nanoalloys with different compositions and configurations, we calculate the mixing energies per atom of 38-atom and 79-atom $\mathrm{Pd}-\mathrm{Ir}$ alloys with increasing Ir composition. As shown in Fig. 5, the stability trend found for 38-TO also applies for 79-TO Pd-Ir nanoclusters. The same configurations of 38-atom and 79-atom Pd-Ir clusters are grouped in the coloured ellipses. Interestingly, for the core-shell group, one can see that the mixing energies of the core-shell structures and the corresponding pure clusters form a straight line for both $\mathrm{Pd}_{\text {shell }} \mathrm{Ir}_{\text {core }}$ and $\mathrm{Pd}_{\text {core }} \mathrm{Ir}_{\text {shell }}$. Since Ir atoms prefer to occupy core sites, when the core sites of the $\mathrm{Pd}_{\text {shell }} \mathrm{Ir}_{\text {core }}$ structure are gradually replaced by Pd atoms, the mixing energy of the core-shell structure increases, until $\Delta=0 \mathrm{eV}$ for the pure Pd clusters. To distinguish the stability of sandwich structures sandwiched by Pd atoms or Ir atoms, we divide them into sandwich-Pd structures (Pd sandwiching Ir) and sandwich-Ir ones (Ir sandwiching Pd). The general stability order for both 38-atom and 79-atom $\mathrm{Pd}-\mathrm{Ir}$ nanoalloys is $\mathrm{Pd}_{\text {shell }} \mathrm{Ir}_{\text {core }}>\mathrm{Pd}_{\text {cup }} \mathrm{Ir}_{\text {ball }}>$ sandwich-Pd $>$ Janus $>$ sandwich-Ir $>$ ordered $\approx \operatorname{Pd}_{\text {ball }} \mathrm{Ir}_{\text {cup }}>\operatorname{Pd}_{\text {core }} \mathrm{Ir}_{\text {shell }}$. Although the mixing energies of the sandwich-type and Janus-type structures range from positive to negative values with increasing Ir proportion, the other arrangements either have all positive or all negative $\Delta$ values, indicating the consistency of the mixing or demixing tendency of a given configuration.

To further measure the stability of Pd-Ir clusters with different compositions and configurations, we calculate the average binding energy of alloy Pd-Ir clusters, as shown in
Fig. 6. It can be clearly observed that the average binding energies of Pd-Ir clusters increase as the proportion of Ir becomes larger, because Ir-Ir and Pd-Ir bonds are stronger than Pd-Pd bonds. From this figure, we can obtain the stability order of Pd-Ir clusters: $\mathrm{Pd}_{\text {core }} \mathrm{Ir}_{\text {shell }}>$ sandwich-Pd $>$ $\mathrm{Pd}_{\text {cup }} \mathrm{Ir}_{\text {ball }}>$ ordered $>\operatorname{Pd}_{\text {ball }} \mathrm{Ir}_{\text {cup }}>\mathrm{Pd}_{\text {core }} \mathrm{Ir}_{\text {shell }}$. By comparing the results of the average binding energy and mixing energy calculations, only the order of the ball-cup and sandwich configurations are reversed.

\subsection{CO adsorption}

To investigate the best adsorption site of a CO molecule on TO 38-atom Pd-Ir nanoalloys and the effect of alloying on CO adsorption strength, we compare the adsorption energies $\left(E_{\text {ads }}\right)$ of $\mathrm{CO}$ on several alloying configurations with pure $\mathrm{Pd}_{38}$ and $\operatorname{Ir}_{38}$ TO clusters in eight surface adsorption sites, as shown in Table 2. As a representative for alloyed clusters, core-shell $\left(\mathrm{Pd}_{32} \mathrm{Ir}_{6}\right.$ and $\left.\mathrm{Pd}_{6} \mathrm{Ir}_{32}\right)$ and Janus $\left(\mathrm{Pd}_{19} \mathrm{Ir}_{19}-\mathrm{Pd}\right.$ and $\left.\mathrm{Pd}_{19} \mathrm{Ir}_{19}-\mathrm{Ir}\right)$ clusters are selected.

For the pure Pd cluster, the preferred position for adsorption of the CO molecule is site 6 (the fcc-hollow on the (111) facet), which has the most negative adsorption energy $-2.12 \mathrm{eV}$, followed by the bridge site between two (111) facets (site 5, $-2.11 \mathrm{eV}$ ). This agrees with a previous study showing that fcc and hep hollow sites are the favoured sites for CO on the extended $\operatorname{Pd}(111)$ surface, and the bridge site is the second most stable. ${ }^{45}$ Our calculations of CO adsorption on extended $\operatorname{Pd}(111), \operatorname{Pd}(100), \operatorname{Ir}(111)$ and $\operatorname{Ir}(100)$ surfaces, also show that hollow sites are preferred on $\operatorname{Pd}(111)$ surfaces with $E_{\text {ads }}=$ $-2.05 \mathrm{eV}$ and $-2.07 \mathrm{eV}$ for fcc hollow and hep hollow sites, respectively, as shown in Table 3 . For the $\operatorname{Pd}(100)$ surface, the bridge-adsorption site is energetically most favourable for CO adsorption, in agreement with CO previous study. ${ }^{46}$ The adsorption on the bridge site on the (111) facet (site 3) of the pure Pd cluster also has a reported adsorption energy of $-2.12 \mathrm{eV}$, since $\mathrm{CO}$ adsorption on the (111)-bridge is unstable

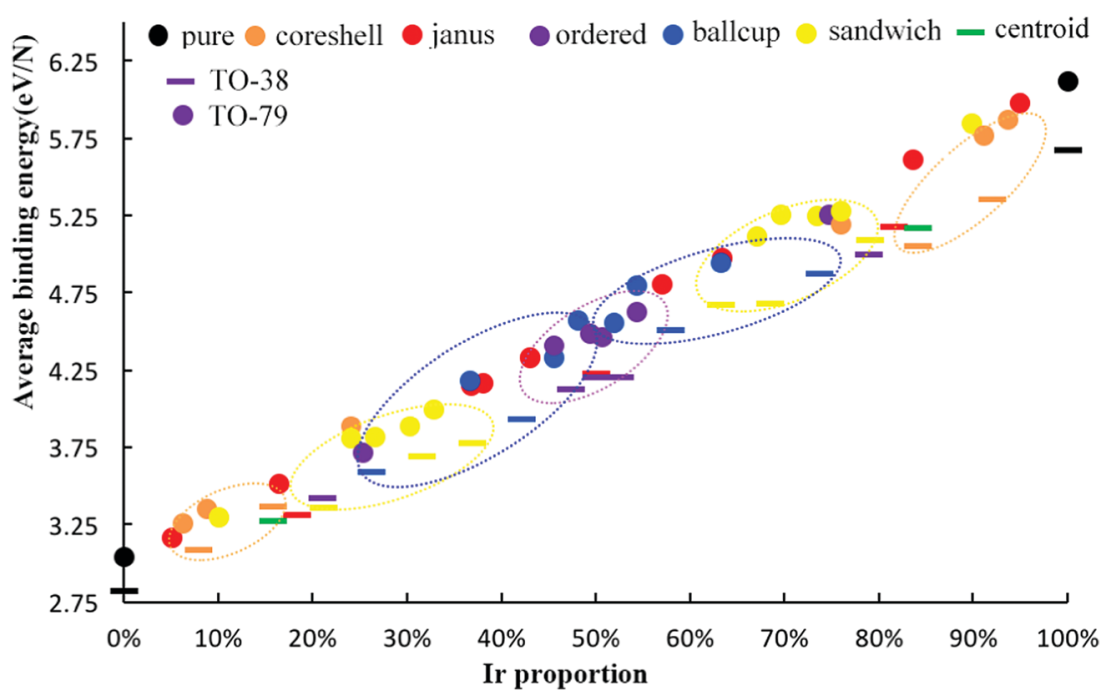

Fig. 6 The average binding energy per atom for 38-TO and 79-TO Pd-Ir nanoalloys with different compositions and configurations. Different colors represent different configurations, the bar symbol denotes the 38-TO Pd-Ir system, and the circle symbol denotes the 79-TO Pd-Ir system. 
Table 2 Adsorption energy ( $E_{\text {ads }} / \mathrm{eV}$ ) of $\mathrm{CO}$ molecule adsorption on 38-atom Pd-Ir nanoalloys. The best site for each structure (the most negative $E_{\text {ads }}$ ) is shown in bold and sites from which the CO relaxes to an alternative site are shown in italics

\begin{tabular}{|c|c|c|c|c|c|c|}
\hline Site & Pure Pd & $\mathrm{Pd}_{\text {shell }} \mathrm{Ir}_{\text {core }}$ & Janus-Pd & Janus-Ir & $\mathrm{Pd}_{\text {core }} \mathrm{Ir}_{\text {shell }}$ & Pure Ir \\
\hline 2 (edge-atop) & -1.64 & -1.67 & -1.69 & -2.59 & -2.75 & -2.58 \\
\hline 5 (111-111 bridge) & -2.11 & -2.03 & -2.01 & -2.28 & -2.41 & -2.25 \\
\hline 6 (fcc-hollow) & -2.12 & -2.05 & -2.16 & -2.75 & -2.06 & -2.24 \\
\hline 7 (hcp-hollow) & -2.02 & -1.97 & -2.14 & -1.99 & -2.06 & -2.44 \\
\hline
\end{tabular}

Table 3 Adsorption energy $\left(E_{\text {ads }} / \mathrm{eV}\right)$ of $\mathrm{CO}$ adsorbed on extended $\mathrm{Pd}$ and Ir surfaces. The best $\mathrm{CO}$ adsorption site for each surface is shown in bold. "-" means there are no four-fold/three-fold hollow sites on the $111 / 100$ extended surfaces

\begin{tabular}{llclc}
\hline Site & $\operatorname{Pd}(111)$ & $\operatorname{Pd}(100)$ & $\operatorname{Ir}(111)$ & $\operatorname{Ir}(100)$ \\
\hline Atop & -1.43 & -1.51 & $-\mathbf{1 . 9 9}$ & $-\mathbf{2 . 2 8}$ \\
Bridge & -2.04 & $-\mathbf{1 . 9 4}$ & -1.65 & -2.12 \\
fcc/hcp-hollow & $-\mathbf{2 . 0 5 / - 2 . 0 7}$ & - & $-1.59 /-1.67$ & - \\
Four-fold-hollow & - & -1.89 & - & -1.74
\end{tabular}

(the site is not a local minimum), and the $\mathrm{CO}$ molecule relaxes to a neighbouring fcc-hollow (site 6).

For the pure $\operatorname{Ir}_{38}$ cluster, the atop site on the edge (site 2) is found to be the favoured position, followed by the atop site in the centre of the (111) facet. This result agrees with the extended Ir surface calculations listed in Table 3, where the atop sites are found to be preferred on both the $\operatorname{Ir}(111)$ and $\operatorname{Ir}(100)$ surfaces. In previous computational and experimental studies of $\mathrm{CO}$ on Ir surfaces, the atop site was reported to be the most favourable site for CO on $\operatorname{Ir}(111)$ and $\operatorname{Ir}(100)$ surfaces. ${ }^{47,48}$ It is worth noting that on the pure Ir cluster, the CO molecule in hollow sites (the (111)-fcc-hollow, (111)-hcp-hollow and (100)fourfold-hollow) all relax to neighbouring bridge positions (site 4 or site 5), probably because the corner atoms of the (111) facet have lower coordination numbers and bind CO more strongly. CO binding on the pure Ir cluster is significantly stronger (with more negative $E_{\text {ads }}$ values) than on the pure Pd cluster for all adsorption sites. Comparing the adsorption energies for the most strongly binding sites for $\mathrm{Ir}_{38}$ and $\mathrm{Pd}_{38}$, $E_{\text {ads }}$ is $0.46 \mathrm{eV}$ lower for $\mathrm{Ir}_{38}$ than $\mathrm{Pd}_{38}$.

Moving to Pd-Ir nanoalloys, for the core-shell type, the fcc-hollow position (site 6) of CO on the $\mathrm{Pd}_{\text {shell }} \mathrm{Ir}_{\text {core }}\left(\mathrm{Pd}_{32} \mathrm{Ir}_{6}\right)$ cluster is found to be the preferred site, as for the pure Pd cluster, followed by the bridge position between two (111) facets (site 5). The adsorption strength of $\mathrm{CO}$ on the $\mathrm{Pd}_{\text {shell }} \mathrm{Ir}_{\text {core }}$ cluster is lower than on the pure Pd cluster, for all sites except the atop sites. For the $\mathrm{Pd}_{\text {core }} \mathrm{Ir}_{\text {shell }}\left(\mathrm{Pd}_{6} \mathrm{Ir}_{32}\right)$ cluster, the edge atop site (site 2) is found to be the most favourable, as for the pure Ir cluster. For almost all sites, the adsorption strength of $\mathrm{CO}$ on $\mathrm{Pd}_{\text {core }} \mathrm{Ir}_{\text {shell }}$ is stronger than on the pure Ir cluster, except for those sites where $\mathrm{CO}$ relaxes to other sites. The difference in the adsorption energies for the best sites on the $\mathrm{Pd}_{\text {core }} \mathrm{Ir}_{\text {shell }}$ and $\mathrm{Pd}_{\text {shell }} \mathrm{Ir}_{\text {core }}$ clusters is greater $(0.70 \mathrm{eV})$ than for the pure metal clusters $(0.46 \mathrm{eV})$, reflecting the strengthening of Ir-CO and weakening of Pd-CO binding on going from the pure to the core-shell clusters.

For Janus structures $\left(\mathrm{Pd}_{19} \mathrm{Ir}_{19}\right)$, the underlying Pd or Ir layers again affect the adsorption strength of $\mathrm{CO}$ on the other metal, but in a less straightforward way than for the core-shell clusters. As seen in Table 2, the adsorption energies of CO on Janus-Pd (i.e. the Pd part of the Janus cluster) are generally more negative (indicating stronger $\mathrm{Pd}-\mathrm{CO}$ binding). The bridge site between the (111) and (100) facets is the preferred site for CO on Janus-Pd (site 4), different from the pure Pd cluster and $\mathrm{Pd}_{\text {shell }} \mathrm{Ir}_{\text {core }}$ cluster, which favour the fcc-hollow (site 6), though the difference in $E_{\text {ads }}$ is only $0.01 \mathrm{eV}$. The adsorption energies on Janus-Ir (i.e. the Ir part of the Janus cluster) are generally more negative (indicating stronger Ir-CO binding) than for the pure Ir cluster. The strongest binding site (more negative adsorption energy) is the centre-atop position (site 1), in contrast to the pure Ir and $\mathrm{Pd}_{\text {core }} \mathrm{Ir}_{\text {shell }}$ clusters, which favour the edge-atop position (site 2). Again, the adsorption of $\mathrm{CO}$ on Ir is significantly greater than on $\mathrm{Pd}$, with a difference in $E_{\text {ads }}$ of the favoured sites of $0.58 \mathrm{eV}$ (i.e. intermediate between that for pure and core-shell clusters).

From Table 2, we see that the fcc hollow site (site 6) is energetically the most favorable position for $\mathrm{CO}$ adsorption on Pd, except for the Janus-Pd cluster, while the atop site on the edge (site 2) is found to be the most favorable site for Ir, except the Janus-Ir cluster. Therefore, for comparison of the adsorption strength of $\mathrm{CO}$ for several alloying configurations and the pure clusters, we select several Pd-Ir configurations with CO adsorption on sites 2 and 6 as representatives, as shown in Fig. 7. Comparing the pure clusters with core-shell and Janus nanoalloys, we observe that the adsorption strength on one particular metal (Pd or Ir) is affected by the presence of the other metal, whether it is in direct contact with the adsorbing metal atom or not.

For CO adsorption on Pd, the situation is complex. Due to the presence of Ir atoms, the CO adsorption strength on the $\mathrm{Pd}_{\text {shell }} \mathrm{Ir}_{\text {core }}$ cluster decreases ( $E_{\text {ads }}$ becomes less negative) while on the Janus-Pd cluster the adsorption strength is greater (more negative $E_{\text {ads }}$ ) compared to pure $\mathrm{Pd}_{38}$. For CO adsorption on Ir, CO molecules adsorbed on the fcc hollow site (site 6) relax to other positions, but the adsorption strength on both $\mathrm{Pd}_{\text {core }} \mathrm{Ir}_{\text {shell }}$ and Janus-Ir clusters still increases compared to pure $\mathrm{Ir}_{38}$, indicating a positive effect of the underlying $\mathrm{Pd}$ atoms. Comparing these results with previous studies of $\mathrm{CO}$ adsorption on $\mathrm{Au}-\mathrm{Rh}$ nanoalloys ${ }^{45}$ the strengthening of Ir-CO 

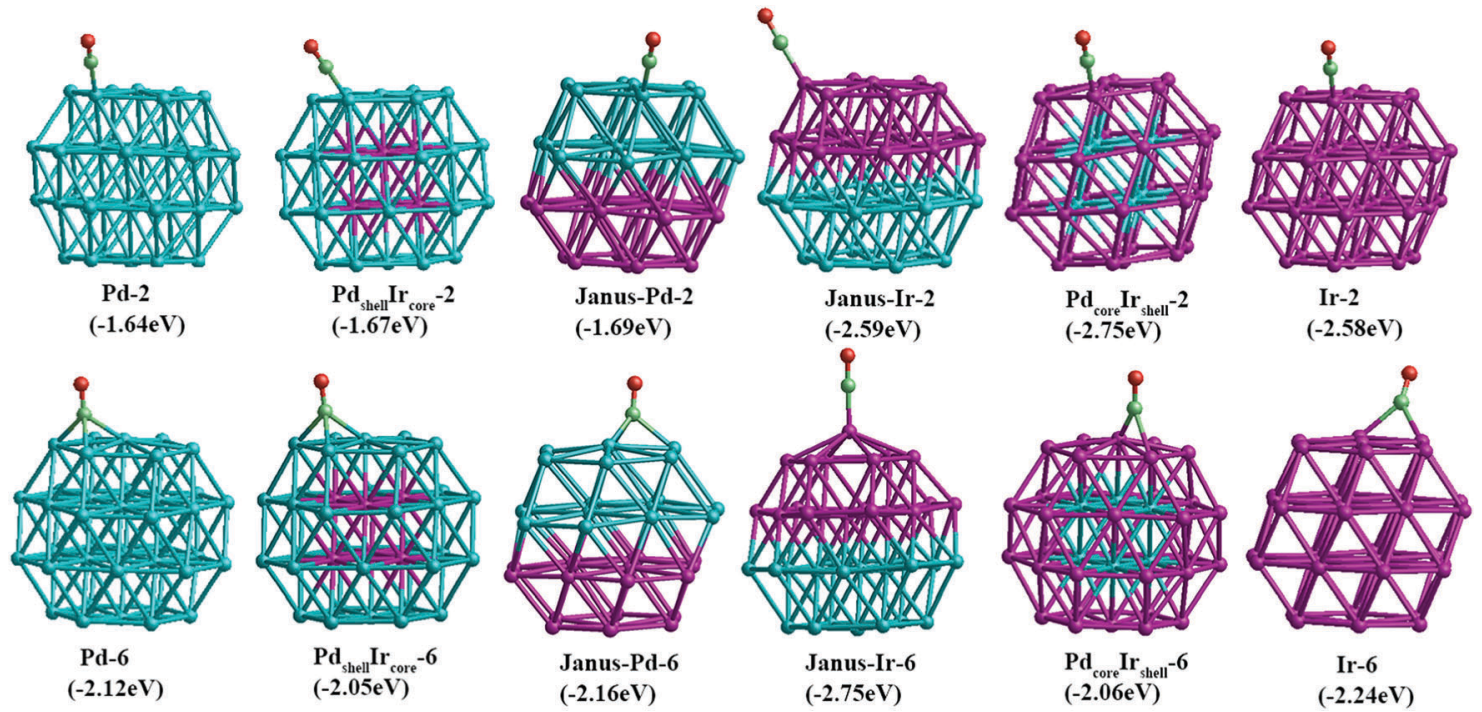

Fig. 7 Adsorption energies and structures of CO on edge-atop (site 2) and fcc hollow (site 6) sites for 38-atom TO pure Pd and Ir cluster and core-shell and Janus Pd-Ir nanoalloys.

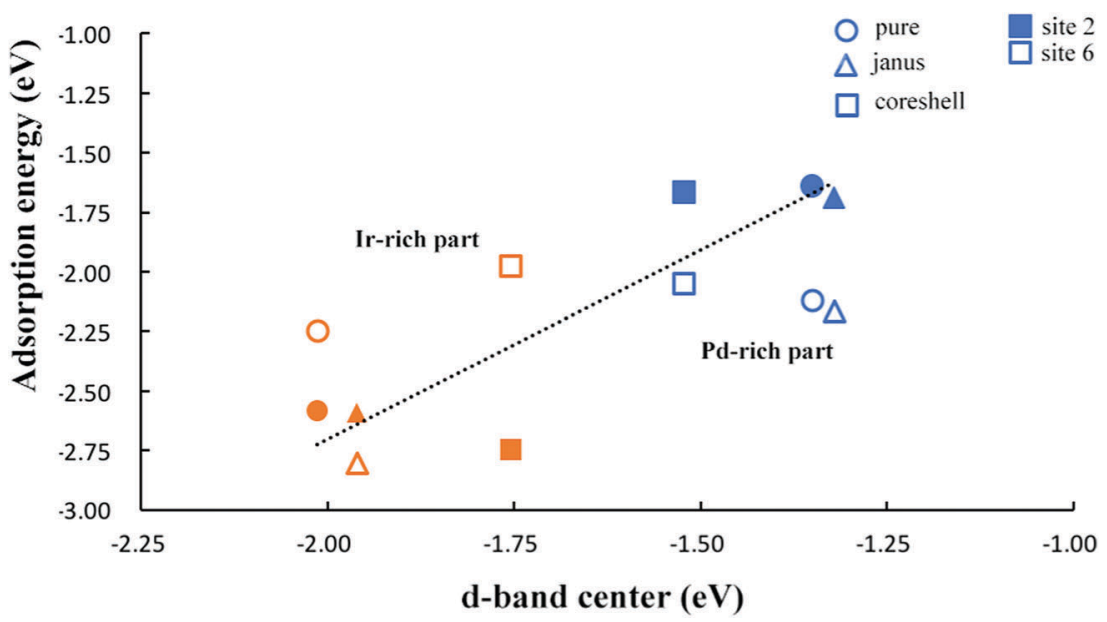

Fig. 8 Plot of d-band center against adsorption energy $\left(E_{\text {ads }}\right)$ for $\mathrm{CO}$ molecular adsorption on Pd-Ir nanoalloys. Different shapes represent different configurations. The light blue color denotes CO on Pd and the orange color denotes CO on Ir. CO on sites 2 and 6 are shown as shaded and empty symbols, respectively.

binding in the presence of Pd and the weakening of Pd-CO binding in the presence of $\mathrm{Ir}$ as seen for the $\mathrm{Pd}_{\text {shell }} \mathrm{Ir}_{\text {core }}$ cluster, could be attributed to a mechanical (strain) effect, whereby (as the metal-metal bond strengths are in the order Ir-Ir > Ir-Pd > Pd-Pd) underlying Ir atoms impose greater rigidity to the $\mathrm{Pd}$ atoms, decreasing the $\mathrm{Pd}-\mathrm{CO}$ binding. Conversely, underlying $\mathrm{Pd}$ atoms reduce the rigidity of the $\mathrm{Ir}$ atoms, allowing stronger Ir-CO binding. However, this argument doesn't apply for all binding sites, and, in particular, does not apply to Janus-Pd, where the presence of Ir leads to an increase in $\mathrm{Pd}-\mathrm{CO}$ binding strength (more negative $E_{\text {ads }}$ ). Therefore, electronic effects must also be investigated.

\subsection{Alloying effect on adsorption strength}

To further investigate trends in the adsorption strength of $\mathrm{CO}$ molecular adsorption on pure clusters and Pd-Ir nanoalloys, we calculate the d-band centers of several Pd-Ir configurations. Fig. 8, shows the relationship between $\mathrm{CO}$ adsorption energies and the d-band centers of the clusters. According to the definition of the d-band model, an upshift in the d-band center (less negative value) is expected to correspond to stronger CO adsorption strength (more negative $E_{\text {ads }}$ ). For adsorption of CO on Pd, it follows the relationship of d-band center and adsorption strength by comparing the d-band centers of $\mathrm{Pd}_{\text {shell }} \mathrm{Ir}_{\text {core }}$ and Janus-Pd clusters with pure $\mathrm{Pd}_{38}$. Since we can clearly observe a significant downshift in the d-band centre for $\mathrm{Pd}_{\text {shell }} \mathrm{Ir}_{\text {core }}$ and a small upshift for Janus-Pd, which corresponds to weaker $\mathrm{CO}$ adsorption on $\mathrm{Pd}_{\text {shell }} \mathrm{Ir}_{\text {core }}$ and stronger adsorption on Janus-Pd, compared to $\mathrm{Pd}_{38}$.

For CO adsorption on Ir, we observe an upshift of the d-band centers for both Janus-Ir and $\mathrm{Pd}_{\text {core }} \mathrm{Ir}_{\text {shell }}$ clusters, when compared with pure $\operatorname{Ir}_{38}$, for both sites, though the upshift for 
Table 4 The d-band centers (eV) and layer charges (|e|) of bare TO 38-atom Pd-Ir clusters, together with charge transfer (|el) to CO, C-O and M-CO distances (Å), and adsorption energies (eV) on site 2 (edge-atop) and site 6 (fcc-hollow) of CO on Pd-Ir clusters

\begin{tabular}{|c|c|c|c|c|c|c|}
\hline Cluster & $d$-Center & Layer charge & Charge transfer & $d_{\mathrm{C}-\mathrm{O}}$ & $d_{\mathrm{M}-\mathrm{C}}$ & $E_{\text {ads }}$ \\
\hline Pure-Pd & -1.351 & 0.183 & -0.413 & 1.162 & 1.842 & -1.641 \\
\hline $\mathrm{Pd}_{\text {shell }} \mathrm{Ir}_{\text {core }}$ & -1.522 & 0.201 & -0.414 & 1.163 & 1.854 & -1.667 \\
\hline Janus-Ir & -1.960 & 0.075 & -0.598 & 1.173 & 1.838 & -2.593 \\
\hline $\mathrm{Pd}_{\text {core }} \mathrm{Ir}_{\text {shell }}$ & -1.755 & 0.270 & -0.577 & 1.171 & 1.839 & -2.747 \\
\hline Pure-Ir & -2.014 & 0.448 & -0.551 & 1.172 & 1.839 & -2.584 \\
\hline Pure-Pd & -1.351 & 0.183 & -0.589 & 1.198 & $2.081,2.037,2.036$ & -2.118 \\
\hline $\mathrm{Pd}_{\text {shell }} \mathrm{Ir}_{\text {core }}$ & -1.522 & 0.201 & -0.581 & 1.197 & $2.051,2.054,2.088$ & -2.050 \\
\hline Janus-Pd & -1.320 & 0.314 & -0.590 & 1.2 & $2.052,2.033,2.052$ & -2.164 \\
\hline Janus-Ir & -1.960 & 0.075 & -0.608 & 1.174 & 1.801 & -2.750 \\
\hline $\mathrm{Pd}_{\text {core }} \mathrm{Ir}_{\text {shell }}$ & -1.755 & 0.270 & -0.665 & 1.191 & $2.043,2.065$ & -2.063 \\
\hline Pure-Ir & -2.014 & 0.448 & -0.646 & 1.193 & $2.026,2.026$ & -2.244 \\
\hline
\end{tabular}

Janus-Ir is small. This follows the change of adsorption strength, since the adsorption of $\mathrm{CO}$ on Ir is stronger (more negative $E_{\text {ads }}$ ) for all alloy configurations on site 2 compared to pure $\mathrm{Ir}_{38}$, consistent with the prediction of the d-band model. However, for $\mathrm{CO}$ adsorption on site 6, the Ir-rich clusters do not always follow the trend of d-band center and adsorption strength, because $\mathrm{CO}$ adsorption on site 6 of Ir-rich clusters is unstable, CO moving to other sites. Fig. 8 also shows that the adsorption strength on site 6 is stronger than on site 2 for Pd-CO binding, while for Ir-CO binding, the adsorption strength on site 2 is not always better than on site 6 since CO on hollow sites is generally not stable, relaxing to neighboring sites (e.g. bridging sites), as can be seen in Fig. 7.

To analyze the charge effect on the adsorption strength of alloy clusters relative to their corresponding pure clusters, we calculate the charges on the (111) layer of bare clusters and charge transfer between clusters and the $\mathrm{CO}$ adsorbate. These, along with the d-band centers and the metal- $\mathrm{CO}$ and $\mathrm{C}-\mathrm{O}$ distances are shown in Table 4. The Pd layer charges for $\mathrm{Pd}_{\text {shell }} \mathrm{Ir}_{\text {core }}$ and Janus-Pd clusters are more positive than for the pure Pd cluster due to the effect of alloying with Ir. Conversely, the Ir layer charges become less positive when alloyed with Pd, though the electronegativity of Ir is equal to Pd (having the value 2.20). Meanwhile, the charge transferred from Janus-Ir and $\mathrm{Pd}_{\text {core }} \mathrm{Ir}_{\text {shell }}$ clusters $\mathrm{CO}$ adsorbed on site 2 is more negative than for the pure Ir cluster. Perhaps, as Ir has fewer d electrons than Pd, this leads to d electron transfer from Pd to Ir.

\section{Conclusions}

The mixing properties of bare Pd-Ir clusters, the adsorption effect and preferred adsorption sites of the $\mathrm{CO}$ molecule on 38-atom Pd-Ir nanoclusters have been investigated theoretically using DFT methods. In agreement with the lower surface and cohesive energy of Pd than Ir, the calculations show that $\mathrm{Pd}_{\text {shell }} \mathrm{Ir}_{\text {core }}$ clusters are the most stable structures and $\mathrm{Pd}_{\text {cup }} \mathrm{Ir}_{\text {ball }}$ clusters are the second most stable, with negative mixing energies for all considered isomers, while $\mathrm{Pd}_{\text {core }} \mathrm{Ir}_{\text {shell }}$ is the highest energy configuration, with large positive mixing energies. These results are also consistent with the site preference for Pd and Ir dopants in the TO Pd-Ir nanoalloys. The general stability order for both 38-atom and 79-atom Pd-Ir nanoclusters is $\mathrm{Pd}_{\text {shell }} \mathrm{Ir}_{\text {core }}>\mathrm{Pd}_{\text {cup }} \mathrm{Ir}_{\text {ball }}>$ sandwich-Pd $>$ Janus $>$ sandwich-Ir $>$ ordered $\approx \mathrm{Pd}_{\text {ball }} \mathrm{Ir}_{\text {cup }}>\mathrm{Pd}_{\text {core }} \mathrm{Ir}_{\text {shell }}$. Moreover, the Ir atoms exhibit significantly stronger adsorption of CO molecules than Pd atoms. The preferred binding site of $\mathrm{CO}$ on the pure Pd cluster is the fcc-hollow on the (111) facet, while the atop site on the edge atom between (111) and (100) facets is found to be the most favorable position for $\mathrm{CO}$ on the pure Ir cluster. These results agree with the favored binding sites for $\mathrm{CO}$ on extended Pd and Ir surfaces. In addition, the d-band center of the pure and nanoalloy clusters has been calculated to understand the adsorption strength of CO molecules on different clusters. Adsorption strength is found to follow the position of the d-band center in most, but not all cases. Charge effects have also been studied, correlating with the increase or decrease of adsorption strength, but the possible role of mechanical (strain) effects remains to be determined.

In future studies, we will investigate the effect of oxide supports (such as $\mathrm{Al}_{2} \mathrm{O}_{3}$ ) on the structures and stabilities of Pd-Ir nanoalloys and on the adsorption of $\mathrm{CO}$ and other molecules, as well as reactions between adsorbed molecules.

\section{Conflicts of interest}

There are no conflicts to declare.

\section{Acknowledgements}

RLJ is grateful to Dr Laurent Piccolo (University of Lyon 1, France) for useful discussions. This work was funded by the Graduate School of Xiamen University (T.-E Fan). H. A. Hussein is grateful to the University of Kufa and the Ministry of Higher Education and Scientific Research (Iraq) for the award of a $\mathrm{PhD}$ scholarship. Calculations were performed on the following HPC facilities: The University of Birmingham BlueBear facility (http://www.bear.bham.ac.uk/bluebear) and the UK's national 
HPC facility, ARCHER, via membership of the UK's HPC Materials Chemistry Consortium, which is funded by EPSRC (EP/L000202), and the EPSRC Critical Mass Grant (EP/J010804/1) TOUCAN.

\section{References}

1 R. W. Murray, Chem. Rev., 2008, 108, 2688.

2 B. Hammerand and J. K. Norskov, Nature, 1995, 376, 238.

3 K. Persson, A. Ersson, K. Jansson, N. Iverlund and S. Jaras, J. Catal., 2005, 231, 139.

4 A. S. Rocha, E. L. Moreno, G. P. M. da Silva, J. L. Zotin and A. C. Faro Jr., Catal. Today, 2008, 133, 394.

5 S. Y. Shen, T. S. Zhao and J. B. Xu, Electrochim. Acta, 2010, 55, 9179.

6 Y. M. Lopez-De Jesús, C. E. Johnson, J. R. Monnier and C. T. Williams, Top. Catal., 2010, 53, 1132.

7 F. Morfin, S. Nassreddine, J. L. Rousset and L. Piccolo, ACS Catal., 2012, 2, 2161.

8 C. Zlotea, F. Morfin, T. S. Nguyen, N. T. Nguyen, J. Nelayah, C. Ricolleau, M. Latroche and L. Piccolo, Nanoscale, 2014, 6, 9955.

9 L. Piccolo, S. Nassreddine, M. Aouine, C. Ulhaq and C. Geantet, J. Catal., 2012, 292, 173.

10 F. Morfin, S. Nassreddine, J. L. Rousset and L. Piccolo, ACS Catal., 2012, 2, 2161.

11 L. Piccolo, S. Nassreddine, G. Toussaint and C. Geantet, ChemSusChem, 2012, 5, 1717.

12 P. E. A. Turchi, V. Drchal and J. Kudrnovský, Phys. Rev. B: Condens. Matter Mater. Phys., 2006, 74, 064202.

13 B. Kolb, S. Müller, D. B. Botts and G. L. W. Hart, Phys. Rev. B: Condens. Matter Mater. Phys., 2006, 74, 144206.

14 R. Ferrando, J. Jellinek and R. L. Johnston, Chem. Rev., 2008, 108, 845.

15 S. Alayoglu, A. U. Nilekar, M. Mavrikakis and B. Eichhorn, Nat. Mater., 2008, 7, 333.

16 L. Kesavan, R. Tiruvalam, M. H. Ab Rahim, M. I. Bin Saiman, D. I. Enache, R. L. Jenkins, N. Dimitratos, J. A. Lopez-Sanchez, S. H. Taylor, D. W. Knight, C. J. Kiely and G. J. Hutchings, Science, 2011, 331, 195.

17 S. Khanal, N. Bhattarai, J. J. Velazquez-Salazar, D. Bahena, G. Soldano, A. Ponce, M. M. Mariscal, S. Mejia-Rosalesc and M. Jose-Yacaman, Nanoscale, 2013, 5, 12456.

18 K. J. Andersson, F. Calle-Vallejo, J. Rossmeisl and I. Chorkendorff, J. Am. Chem. Soc., 2009, 131, 2404.

19 F. Tao, M. E. Grass, Y. Zhang, D. R. Butcher, J. R. Renzas, Z. Liu, J. Y. Chung, B. S. Mun, M. Salmeron and G. A. Somorjai, Science, 2008, 322, 932.

20 F. Tao, M. E. Grass, Y. Zhang, D. R. Butcher, F. Aksoy, S. Aloni, V. Altoe, S. Alayoglu, J. R. Renzas, C. Tsung, Z. Zhu, Z. Liu, M. Salmeron and G. A. Somorjai, J. Am. Chem. Soc., 2010, 132, 8697.

21 V. Soto-Verdugo and H. Metiu, Surf. Sci., 2007, 601, 5332.
22 A. Dhouib and H. Guesmi, Chem. Phys. Lett., 2012, 521, 98.

23 G. Ertl, M. Neumann and K. M. Streit, Surf. Sci., 1977, 64, 393.

24 O. Cairon and H. Guesmi, Phys. Chem. Chem. Phys., 2011, 13, 11430.

25 W. Bouderbala, A.-G. Boudjahem and A. Soltani, Mol. Phys., 2014, 112, 1789.

26 J. B. A. Davis, S. L. Horswell, L. Piccolo and R. L. Johnston, J. Organomet. Chem., 2015, 792, 190.

27 J. B. A. Davis, S. L. Horswell and R. L. Johnston, J. Phys. Chem. A, 2013, 118, 208.

28 J. B. A. Davis, R. L. Johnston, L. Rubinovich and M. Polak, J. Chem. Phys., 2014, 141, 224307.

29 T. H. Andriamiharintsoa, A. Rakotomahevitra, L. Piccolo and C. Goyhenex, J. Nanopart. Res., 2015, 17, 217.

30 B. Coq and F. J. Figueras, J. Mol. Catal. A: Chem., 2001, 173, 117.

31 L. L. Wang and D. D. Johnson, J. Am. Chem. Soc., 2009, 131, 14023.

32 Y. Sun, B. Wiley, Z. Y. Li and Y. Xia, J. Am. Chem. Soc., 2004, 126, 9399.

33 F. Baletto, C. Mottet and R. Ferrando, Phys. Rev. Lett., 2003, 90, 135504.

34 D. Cheng, W. Wang, S. Huang and D. Cao, J. Phys. Chem. C, 2008, 112, 4855.

35 L. O. Paz-Borbon, A. Gupta and R. L. Johnston, J. Mater. Chem., 2008, 18, 4154.

36 G. Kresse and J. Hafner, Phys. Rev. B: Condens. Matter Mater. Phys., 1993, 47, 558.

37 P. E. Blöchl, Phys. Rev. B: Condens. Matter Mater. Phys., 1994, 50, 17953.

38 G. Kresse and D. Joubert, Phys. Rev. B: Condens. Matter Mater. Phys., 1999, 59, 1758.

39 J. P. Perdew, K. Burke and M. Ernzerhof, Phys. Rev. Lett., 1996, 77, 3865.

40 R. Ferrando, A. Fortunelli and G. Rossi, Phys. Rev. B: Condens. Matter Mater. Phys., 2005, 72, 085449.

41 I. Demiroglu, Z. Y. Li, L. Piccolo and R. L. Johnston, Catal. Sci. Technol., 2016, 6, 6916.

42 V. Rosato, M. Guillope and B. Legrand, Philos. Mag. A, 1989, 59, 321.

43 J. E. Bercaw, A. C. Durrell, H. B. Gray, J. C. Green, N. Hazari, J. A. Labinger and J. R. Winkler, Inorg. Chem., 2010, 49, 1801.

44 L. Vitos, A. V. Ruban, H. L. Skriver and J. Kollar, Surf. Sci., 1998, 411, 186.

45 D. Loffreda, D. Simon and P. Sautet, Surf. Sci., 1999, 425, 68. 46 A. Eichler and J. Hafner, Phys. Rev. B: Condens. Matter Mater. Phys., 1998, 57, 10110.

47 W. P. Krekelberg, J. Greeley and M. Mavrikakis, J. Phys. Chem. B, 2004, 108, 987.

48 I. A. Erikat, B. A. Hamad and J. M. Khalifeh, Eur. Phys. J. B, 2009, 67, 35 . 